\title{
Time error in lifted weights as affected by presentation order and judgment mode
}

\author{
BURRTON WOODRUFF, DENNIS L. JENNINGS, and NICHOLAS L. RICO \\ Butler University, Indianapolis, Indiana 46208
}

\begin{abstract}
In the method of constant stimulus differences, results obtained under two presentation orders (standard first and standard second) are commonly combined to eliminate time error. The present study shows that this technique is not adequate for controlling time error as the presentation orders have differential effects upon PSE. Using a two-handed technique, 96 subjects judged lifted weights on the comparative rating scale. The subjects lifted the standard before, with, or after the variable stimulus. They also either judged the standard in terms of the variable or the variable in terms of the standard (mode of judgment). Judgment mode, a previously uninvestigated confounding variable of presentation order was not an important factor in influencing the value of the PSE.
\end{abstract}

In the method of constant stimulus differences, the observer is asked to judge if one member of a pair of stimuli is greater or less than the other member. One of the stimuli is the standard stimulus and the other is selected from a set of comparison stimuli. Each comparison stimulus is paired with the standard stimulus many times. From the judgments, a number of measures can be calculated. The point of subjective equality (PSE) specifies the value of the comparison stimulus which would be judged equal to the standard. Typically, the values of the PSE and standard stimulus are different and this difference is called the constant error.

Since the pair of stimuli are separated in space or time, side effects of this separation can influence the judgments made by the observer. Space error is the aspect of constant error specifically related to changes in judgment caused by spatial separation. Space error is usually easily controlled by either counterbalancing conditions of presentation or by eliminating the spatial variable entirely. Time error (Fechner, $1860 / 1966$ ) has to do with the influence on judgment of experiencing one stimulus before the other. Traditionally, a positive time error indicates underestimation of the second stimulus (PSE is greater than the standard); a negative time error indicates overestimation of the second stimulus (PSE is less than the standard) (Needham, 1934). Fechner suggested controlling for time error by using two presentation orders. For half the trials, present the standard stimulus first ( $S_{1}$ presentation order), and in the other trials present the standard stimulus second $\left(S_{2}\right.$ presentation order). In this manner, the under- or overestimation of the second stimulus will affect both

This research was supported in part by the Faculty Fellowship Program of Butler University. The authors acknowledge and thank Pam Powell Shackelford for running a pilot study. the standard and comparison or variable stimuli equally. ${ }^{1}$

However, there is no reason that the variable and standard stimuli have to be equally affected by time error in the two presentation orders. In adaptation-level theory (Helson, 1964), the roles of the standard and variable are different, and consequently the presentation orders could well affect the two differentially. The Michels and Helson (1954) quantitative model of the effect of presentation order on PSE specifically allows for a differential effect as the usual state. Probably because presentation orders were a logical experimental control, little work has been done with them per se. For example, Michaels and Helson (1954) do not cite any attempts in the literature to investigate any differential effects. An additional problem is that there hasn't been any convenient, meaningful baseline to determine how large an effect the presentation orders have. In lifted weights, almost always the PSE for the $S_{1}$ presentation order is less than that for the $S_{2}$ presentation order. But this knowledge doesn't allow conclusions of differential effects to be drawn.

The present study used a two-handed weight-lifting procedure to provide a baseline to assess the relative effects of the presentation orders on judgment. When using two hands, the subjects can be presented with both stimuli simultaneously as well as successively. This allows for a direct assessment of the size of the time errors.

In addition, there is an asymmetry in the procedure most commonly used in the method of constant stimulus differences. The subject always judges if the second stimulus is greater or less than the first stimulus (Guilford, 1954). This means that for the $S_{1}$ presentation order the variable stimulus is judged in terms of the standard stimulus. In the $S_{2}$ presentation order, the standard stimulus is judged with reference 
to the variable stimulus given on that trial. That is, there are two judgment modes in addition to the two presentation orders. The subject can judge the variable in terms of the standard ( $\mathrm{S}$ mode) or he can judge the standard in terms of the variable (V mode). The usual determination uses the $S$ mode with the $S_{1}$ presentation order and the $\mathrm{V}$ mode with the $\mathbf{S}_{\mathbf{2}}$ presentation order.

The remaining combinations of presentation order and judgment mode can be obtained by requiring the subject to judge the first stimulus in terms of the second stimulus. Now, with the $S_{2}$ presentation order, the variable is judged in terms of the standard ( $S$ mode). Likewise, the $V$ mode is used for the $S_{1}$ presentation order when the subject judges the first stimulus in terms of the second.

Briefly, then, in this study there are two major independent variables being manipulated. One is presentation order (standard first, $S_{1}$; standard second, $S_{2}$; stand ard and variable simultaneous, $S_{0}$ ). The other is judgment mode (use of the standard as the reference, $S$ mode; use of the variable as the reference, $\mathrm{V}$ mode). Together, these variables determine whether the subject judges the second stimulus in terms of the first or the first stimulus in terms of the second.

The actual experiment was run using the comparative rating scale modification of the method of constant stimulus differences (Helson, Michels, \& Sturgeon, 1954). This modification produces highly reliable judgment functions with many fewer stimulus presentations than does the classical method.

\section{METHOD}

\section{Subjects}

The subjects were 96 right-handed Butler University undergraduates. The median age was 20.0 years. Half of the subjects were female.

\section{Apparatus}

The weights were $5 \times 2 \times 2$ in. $(12.7 \times 5.1 \times 5.1 \mathrm{~cm})$ prescription bottles weighted with lead shot and cotton to the specified masses. The standard stimulus was $300 \mathrm{~g}$. The five series stimuli ranged from 200 to $400 \mathrm{~g}$ in $50-\mathrm{g}$ steps.

During the experiment, the subject and the experimenter were seated across a 38 -in. $(.96 \mathrm{~m})$ wide table. A 32-in. $(.81 \mathrm{~m})$ high blind at the center of the table prevented the subject from seeing any part of the experimenter's side of the table. At the bottom, the blind had two holes, $13 \mathrm{in.}(.33 \mathrm{~m})$ apart, cut in it, through which the subject inserted his hands. Cloth hanging from the top of the openings prevented the subject from seeing any of the stimulus presentation procedure.

The weights rested on a foam pad and when, placed between the subject's fingers for lifting, rested on a second foam pad.

\section{Design}

Basically, the experiment was a 3 by $2^{4}$ factorial arrangement of treatments with two replications. The three-valued factor was simultaneous presentation of the pair vs. successive presentation where the second stimulus was judged against the first vs. successive presentation where the first stimulus was judged against the second. Three of the four two-valued factors were experimenter (D.L.J. and
N.L.R.), sex of the subject, and the hand that lifted the standard stimulus.

The fourth two-valued factor was whether the subject judged the weight in his right hand in terms of the one in his left, or vice versa. For the simultaneous presentations, this last variable determined the judgment mode. If the standard stimulus was in the subject's right hand and he was to judge the left stimulus in terms of the right, then this would be the $S$ mode. If he was to judge right in terms of left, it would be the $V$ mode.

For the successive conditions, this last factor also determined which hand would first lift a weight. If the subject was to judge the second weight in terms of the first and the standard was in the right hand and if he was instructed to judge the right weight in terms of the left, then the left weight or variable had to be lifted first. If the subject was judging the first weight in terms of the second and the standard was again in the right hand, then to judge the right weight in terms of the left, the subject had to lift the right weight or standard first.

Because presentation order and judgment mode are of primary interest, the results are given in terms of these variables and not in terms of the subject's task. Table 4 includes a summary of the 12 arrangements of the orders of presentation and judgment to show the presentation order and judgment mode specified by each of these arrangements.

\section{Procedure}

Each subject judged 110 pairs of stimuli-10 practice judgments and 100 data judgments-for one of the 12 arrangements of presenting and judging conditions. The 100 data judgments represent 20 judgments of each variable stimulus with the standard stimulus.

Each data sheet was prepared by listing 22 of the 120 permutations of the five series stimuli. That is, in each consecutive block of five trials, each variable was compared with the standard stimulus once. A total of 64 data sheets were prepared-32 for the successive conditions and 32 for the simultaneous condition. The sheets were than assigned randomly to the four stimulus arrangements for one of the successive presentation conditions and to the four arrangements of the simultaneous presentation conditions. The sex of the subject and the experimenter identification were then marked on the sheets in each of these stimulus arrangements. The sheets were then ordered randomly for each experimenter so that one replication of the simultaneous and the first successive presentation condition was completed before the second replication was started.

The data sheets for the successive presentation condition were then duplicated totally for use in the other successive presentation condition, where the first weight lifted was judged in terms of the second. The subjects for this condition were run after an experimenter had completed collecting the data on the other conditions.

When a subject entered the experimental cubicle, he was seated facing the blind and was read a set of instructions concerning his task. He was told that this was an experiment in weight discrimination and that there were no right or wrong answers. The subject was to report how the pairs of weights felt to him. He would lift two weights and then judge how one felt in terms of the other, using the following set of categories: very much heavier (9), much heavier (8), heavier (7), little heavier (6), equal (5), little lighter (4), lighter (3), much lighter (2), and very much lighter (1). The subject was also told that he could add categories to the top or bottom of the scale if he wished. The numbers in parentheses indicate the values assigned to each judgment for purposes of analysis and were not disclosed to the subject.

Then the experimenter demonstrated how to lift each weight. The subject was to rest his hands comfortably on their sides. The weights were then placed between the thumb and fingers. The ends of the fingers and thumb could then grasp the weight and lift it, using a wrist movement. The forearm was not to be raised from the table, and nor were the weights to be jiggled. 
The remainder of the instructions specilied, if appropriate, which hand the subject was to lift tirst and whether he was to judge the weight in his right hand in terms of the one in his left, or vice versa. During the experiment, the subject had in front of him a listing of the judgment categorics and a concise statement of his judgment task. e.g. " "remember: how much heavier or lighter is the left one."

In the successive conditions. the first weight was placed in one hand. lifted, and then removed betore the second weight was placed in the other hand. The subject could make his judgment any time after he had lifted the second weight. The interval between the members of the pair was roughly $2.4 \mathrm{sec}$. For the simultaneous condition, the weights were placed in the subject's hands at the same time. The interval between the pair of weights being judged depended upon when the subject made his judgment. The interval between successive pairs could be as short as about 5 sec.

\section{RESULTS AND DISCUSSION}

For each subject, the mean judgment for each comparison stimulus was calculated and the PSE or adaptation level was obtained using a least squares solution to find the stimulus to which the subject would have given a judgment of equal (5.0).

The judgment functions under various of the 12 stimulus arrangements differed enough in form to make it impossible to use only one least squares model to obtain an accurate value for PSE. Consequently, the calculations were done three times. A least squares estimate of PSE was obtained using the Helson-Himmelstein model (Helson \& Himmelstein, 1955), the reformulated Fechner law model (Michels \& Helson, 1949), and the unadorned straight line model. The standard error of estimate was calculated in every case, and the value of PSE derived from the method with the smallest error of prediction was used in subsequent analyses.

Table 1 lists the mean PSE for each of the 12 combinations of presentation order and judgment mode. Table 2 is the source table of the five-way analysis of variance used to assess the reliabilities of the differences in the PSEs.

\section{Presentation Order}

The $S_{1}$ presentation order had a mean PSE of $284 \mathrm{~g}$; the $S_{0}$ presentations had a mean value of $316 \mathrm{~g}$; and the $S_{2}$ presentation order had a mean PSE of $329 \mathrm{~g}$. Pairwise comparisons of these means by Scheffé's test (McNemar, 1969) shows all differences to be reliable $\left[S_{1}\right.$ and $S_{2}: t(48)=9.157, p<.001 ; S_{1}$ and $S_{0}: t(48)=6.482 . p<.001 ; S_{0}$ and $S_{2}: t(48)=$ 2.675. $p<.0201$. That the $S_{1}$ presentation order yielded a smaller PSE is typical and expected. The intermediate value of the PSE for $S_{O}$ is in line with the value expected when the time error is taken into consideration. The difference in PSEs of the $S_{1}$ and $S_{0}$ conditions $(32 \mathrm{~g})$ is 2.5 times larger than that difference for the $S_{O}$ and $S_{2}$ conditions $(13 \mathrm{~g})$. This difference is reliable $(p<.025)$, as evidenced by the orthogonal contrast (Edwards, 1964) in Table 2. which compares the sum of the PSEs of $S_{1}$ and $S_{2}$ against twice the value for $S_{O}$. This comparison shows that the arithmetic average of the PSEs of $S_{1}$ and $S_{2}$ is different from the PSE of $S_{0}$. This could have resulted only because the value for $S_{0}$ is not equidistant from the values for $S_{1}$ and $S_{2}$; hence, the reliable differential effect.

The differential effect is clearly seen in Table 3 . which gives the judgment functions for the $S_{1}, S_{0}$, and $S_{2}$ conditions. The functions were calculated by first summing for each variable the average judgment of all eight subjects in each of the 12 conditions and then finding the mean judgment. All judgments not already so were then converted into the equivalent of $S$-mode judgments. The transformation involves cakculating how different each mean judgment is from the equal judgment (5.0) and then adding or subtracting 5.0 as the case requires. For example, a $V$-mode judgment of 3.5 states that the standard stimulus was judged to be 1.5 categories lighter than the variable stimulus. Therefore. using the $\mathrm{S}$ mode of judgment, the variable should have been judged 1.5 units heavier than the standard (6.5).

Table 3 indicates that the $S_{0}$ and $S_{2}$ mean judgnents were closer together for all stimuli than were the $S_{1}$ and $S_{0}$ judgments. The differential effect of presentation order was consistently present for all of the comparison stimuli. All together, the results show that time error would not be eliminated by combining the results of the two presentation orders. If we assign the over- or underestimation to the

Table 1

Mean PSE (g) and Sample Standard Deviation (g) for the 12 Stimulus Conditions

\begin{tabular}{|c|c|c|c|c|c|c|c|c|c|c|c|c|}
\hline $\begin{array}{l}\text { Judgment } \\
\text { Code* }\end{array}$ & SIL & SIR & $\mathrm{V} 2 \mathrm{R}$ & V2L & S-L & S-R & V-R & V-L & S2L & S2R & VIR & VIL \\
\hline $\begin{array}{l}\text { Condition } \\
\text { Numbert }\end{array}$ & $1 \mathrm{~A}$ & 1B & $5 A$ & $5 B$ & $3 \mathrm{~A}$ & $3 B$ & $4 \Lambda$ & $4 \mathrm{~B}$ & $6 \Lambda$ & $6 \mathrm{~B}$ & $2 \AA$ & $2 \mathrm{~B}$ \\
\hline PSI: & 287 & 281 & 277 & 291 & 312 & 307 & 332 & 312 & 322 & 326 & 320 & 347 \\
\hline SD+t & 154 & 155 & 139 & 164 & 108 & 166 & 275 & 315 & 127 & 208 & 247 & 211 \\
\hline
\end{tabular}

*The first symbol indicates whether the judgment was referenced to the standard (S) or variable (V) stimulus. The second symbol tells whether the reference stimulus was lifted before (1), after (2), or with (-) the comparison stimulus. The third symbol indicates whether the reference stimulus was in the left $(L)$ or the right (R) hand.

tThese numbers were assigned on the basis of the subject's task.

$+t T h e$ values listed here are 10 times the actual value. 
Table 2

Analysis of Variance of the 96 Individual PSEs

\begin{tabular}{|c|c|c|c|c|c|}
\hline & Source & $\mathrm{df}$ & Mean Square & F Ratio & Alpha \\
\hline \multirow[t]{4}{*}{ A } & Presentation Order & 2 & $16,813.052$ & 49.767 & $\mathrm{p}<.001$ \\
\hline & $A_{0} \quad S_{1}$ vs. $S_{2}$ & (1) & $(31,790.890)$ & 94.101 & $\mathrm{p}<.001$ \\
\hline & $A_{1} \quad S_{1}+S_{2}$ vs. $2 S_{0}$ & (1) & $(1,835.213)$ & 5.432 & $\mathrm{p}<.025$ \\
\hline & Judgment Mode & 1 & $1,242.720$ & 3.678 & $\mathrm{p}<.100$ \\
\hline $\mathrm{C}$ & Side of Standard & 1 & 138.240 & .409 & n.s. \\
\hline D & Sex of Subject & 1 & 75.260 & .223 & n.s. \\
\hline $\mathrm{E}$ & Experimenter & 1 & .634 & .002 & {$[\mathrm{p}<.050]$} \\
\hline \multicolumn{2}{|c|}{ A by B } & 2 & 379.755 & 1.124 & n.s. \\
\hline \multicolumn{2}{|c|}{$\mathrm{A}_{0}$ by $\mathrm{B}$} & (1) & $(409.051)$ & 1.210 & n.s. \\
\hline \multicolumn{2}{|c|}{$\mathrm{A}$, by $\mathrm{B}$} & (1) & 350.460 & 1.049 & n.s. \\
\hline \multicolumn{2}{|c|}{ A by C } & 2 & $1,597.595$ & 4.729 & $\mathrm{p}<.025$ \\
\hline \multicolumn{2}{|c|}{$\mathrm{A}_{0}$ by $\mathrm{C}$} & (1) & $(522.122)$ & 1.545 & n.s. \\
\hline \multicolumn{2}{|c|}{$A_{1}$ by $C$} & (1) & $(2,673.068)$ & 7.912 & $\mathrm{p}<.010$ \\
\hline \multicolumn{2}{|c|}{ B by C } & 1 & 637.570 & 1.887 & n.s. \\
\hline \multicolumn{2}{|c|}{ A by $B$ by $C$} & 2 & 890.015 & 2.634 & $\mathrm{p}<.10$ \\
\hline \multicolumn{2}{|c|}{$\mathrm{A}_{0}$ by B by C } & (1) & $(14.631)$ & .043 & n.s. \\
\hline \multicolumn{2}{|c|}{$A_{1}$ by $B$ by $C$} & (1) & $(1,765.400)$ & 5.226 & $\mathrm{p}<.050$ \\
\hline \multicolumn{2}{|c|}{ Pooled Interaction } & 34 & 496.212 & 1.469 & n.s. \\
\hline \multicolumn{2}{|c|}{ Error } & 48 & 337.837 & & \\
\hline \multicolumn{2}{|c|}{ Total } & 95 & & & \\
\hline
\end{tabular}

second-presented stimulus, then for the $S_{1}$ presentation order, the variable stimuli were overestimated by $32 \mathrm{~g}$ with respect to the baseline of the PSE of the $S_{0}$ condition. That is, it took only a 284-g variable stimulus in the $S_{1}$ condition to feel as heavy as a $316-g$ variable in the $S_{O}$ condition. With the $\mathrm{S}_{2}$ presentation order, the standard stimulus was overestimated, but by only $13 \mathrm{~g}$. For the $S_{2}$ presentations, the standard stimulus felt as heavy as a $329-g$ comparison weight. Again, the baseline is the PSE of the $S_{O}$ condition. This experiment showed that when the standard is presented as the second stimulus, it was overestimated less than the comparison stimuli when they were presented second. There are not two separate processes occurring here, as the usual interpretation of one negative and one positive constant error would conclude, but just one-overestimation. The same analysis could, of course, be couched in terms of underestimation alone if the time error were posited as affecting the first stimulus presented instead of the second. Other than the law of parsimony, there is, of course, nothing which prevents one from using a traditional analysis

Table 3

Mean Judgment

Standard of $300 \mathrm{~g}$

\begin{tabular}{cccc}
\hline & \multicolumn{3}{c}{ Presentation Order } \\
\cline { 2 - 4 } $\begin{array}{c}\text { Variable } \\
\text { Stimulus } \\
(\mathrm{g})\end{array}$ & $\begin{array}{c}\text { Standard } \\
\text { First }\end{array}$ & $\begin{array}{c}\text { Simul- } \\
\text { taneous } \\
\left(\mathrm{S}_{0}\right)\end{array}$ & $\begin{array}{c}\text { Standard } \\
\text { Second } \\
\left(\mathrm{S}_{2}\right)\end{array}$ \\
\hline 200 & $\left(\mathrm{~S}_{1}\right)$ & 2.3 & 2.5 \\
250 & 2.7 & 3.6 & 3.4 \\
300 & 4.1 & 4.6 & 4.4 \\
350 & 5.5 & 5.8 & 5.4 \\
400 & 6.7 & 6.8 & 6.3 \\
\hline
\end{tabular}

of the effect into overestimation in one presentation order and underestimation in the other.

One complication to the preceding documentation of a differential effect of the presentation orders is that the $S_{1}$ and $S_{2}$ conditions do not precisely correspond to the classical presentation orders. Each condition has observations with both judgment moces. Classically, the standard-first presentation order has judgments in only the $S$ mode and the standard-second presentation order consists only of $V$-mode judgments. In the present study, the remaining two combinations of these variables were included. Orthogonal and nonorthogonal contrasts allow partitioning the results to examine the size and reliability of the effects of judgment mode in conjunction with the traditional presentation orders. The coefficients for a number of contrasts are detailed in Table 4. Some of the contrasts form orthogonal sets and are included in the analysis of variance source table (Table 2). For standard-first presentations, the mean PSE was not different for the two modes (Contrast 1: $284 \mathrm{~g}$ for both modes). Nor was there a reliable difference between modes of judgment for presentations where the standard was second [Contrast 2: $333 \mathrm{~g}$ for $\mathrm{V}$ mode; $324 \mathrm{~g}$ for $\mathrm{S}$ mode; $\mathrm{F}(1,48)=1.911, \mathrm{p}>.100]$. In addition, there was not a reliable difference between the traditional and nontraditional modes. Incidentally, this contrast also indicates that there is no evidence that judging first in terms of second led to different PSEs than the more usual judging of second in terms of first. The traditional modes are where the subject judged second in terms of first, and the nontraditional are where the subject judged first in terms of second [Contrast 3: $309 \mathrm{~g}$ for traditional modes; $304 \mathrm{~g}$ for nontraditional modes; $F(1,48)=1.069, \mathrm{p}>.100]$. Finally, the 
Table 4

Coefficients for the Orthogonal and Nonorthogonal Contrasts Used in the Analysis of Variance

\begin{tabular}{|c|c|c|c|c|c|c|c|c|c|c|c|c|}
\hline \multirow{4}{*}{$\begin{array}{l}\text { Presentation Order (A) } \\
\text { Side of Standard (C) } \\
\text { Judgment Mode (B)* } \\
\text { Condition Number }\end{array}$} & \multicolumn{4}{|c|}{$S_{1}$} & \multicolumn{4}{|c|}{$S_{0}$} & \multicolumn{4}{|c|}{$\mathrm{S}_{2}$} \\
\hline & \multicolumn{2}{|c|}{ L } & \multicolumn{2}{|c|}{$\mathbf{R}$} & \multicolumn{2}{|c|}{ L } & \multicolumn{2}{|c|}{$\mathbf{R}$} & & \multicolumn{2}{|c|}{$\mathbf{R}$} \\
\hline & $2 S$ & $1 \mathrm{~V}$ & $2 S$ & $1 \mathrm{~V}$ & $-\mathrm{S}$ & $-\mathrm{V}$ & $-\mathrm{S}$ & $-\mathbf{V}$ & $1 S$ & $2 \mathrm{~V}$ & 1S & $2 \mathrm{~V}$ \\
\hline & $1 \mathrm{~A}$ & $5 \mathrm{~A}$ & 1B & $5 \mathrm{~B}$ & $3 \mathbf{A}$ & $4 A$ & 3B & 4B & $6 \mathrm{~A}$ & $2 \mathrm{~A}$ & $6 \mathrm{~B}$ & 2B \\
\hline \multicolumn{13}{|c|}{ s Contained } \\
\hline $\mathbf{A}_{0}$ & -1 & -1 & -1 & -1 & 0 & 0 & 0 & 0 & +1 & +1 & +1 & +1 \\
\hline$A_{1}$ & +1 & +1 & +1 & +1 & -2 & -2 & -2 & -2 & +1 & +1 & +1 & +1 \\
\hline B & -1 & +1 & -1 & +1 & -1 & +1 & -1 & +1 & -1 & +1 & -1 & +1 \\
\hline $\mathrm{C}$ & +1 & +1 & -1 & -1 & +1 & +1 & -1 & -1 & +1 & +1 & -1 & -1 \\
\hline$A_{0}$ by $C$ & -1 & -1 & +1 & +1 & 0 & 0 & 0 & 0 & +1 & +1 & -1 & -1 \\
\hline$A_{1}$ by $C$ & +1 & +1 & -1 & -1 & -2 & -2 & +2 & +2 & +1 & +1 & -1 & -1 \\
\hline$B$ by $C$ & -1 & +1 & +1 & -1 & -1 & +1 & +1 & -1 & -1 & +1 & +1 & -1 \\
\hline$A_{0}$ by $B$ by $C$ & +1 & -1 & -1 & +1 & 0 & 0 & 0 & 0 & -1 & +1 & +1 & -1 \\
\hline$A_{1}$ by $B$ by $C$ & -1 & +1 & +1 & -1 & +2 & -2 & -2 & +2 & -1 & +1 & +1 & -1 \\
\hline \multicolumn{13}{|c|}{ Contrasts Showing the Joint Effect of Presentation Order and Judgment Mode } \\
\hline Contrast 1 & +1 & -1 & +1 & -1 & 0 & 0 & 0 & 0 & 0 & 0 & 0 & 0 \\
\hline Contrast 2 & 0 & 0 & 0 & 0 & 0 & 0 & 0 & 0 & -1 & +1 & -1 & +1 \\
\hline Contrast 3 & +1 & -1 & +1 & -1 & 0 & 0 & 0 & 0 & -1 & +1 & -1 & +1 \\
\hline Contrast 4 & +1 & -1 & +1 & -1 & 0 & 0 & 0 & 0 & +1 & -1 & +1 & -1 \\
\hline
\end{tabular}

*A 2 indicates the second stimulus was judged against the first. A 1 indicates the first was judged against the second. S indicates the $S$ mode; $V$ indicates the $V$ mode.

difference in PSE for the standard-first and standard-second presentation orders for the traditional and nontraditional modes is not reliable. In both instances, the PSE for the $S_{2}$ presentations is larger. The difference in mean PSEs for the traditional mode was $49 \mathrm{~g}$. For the nontraditional presentations, the difference was $40 \mathrm{~g}$ [Contrast 4: $\mathrm{F}(1,48)=0.848, \mathrm{p}<.250]$. Obviously, there is nothing in these results concerning judgment mode which indicates an interactive effect with presentation order.

The base against which time error was measured is the PSE for the $S_{0}$ presentations and not the value of the standard stimulus. The constant error which occurs with psychophysical judgment and is not explanable as time or space error (Helson, 1964) makes this point of physical equality worthless in assessing the size of the differential effect in time error. As it was, the PSE for $S_{o}$ is reliably greater than the value of physical equality $[t(31)=4.014$, $p<.001]$. This could indicate both the phenomenon of decentered judgments and a space error as well. Helson's (1964) prediction equation for PSE for the two traditional presentation orders gives a value of $256 \mathrm{~g}$ for the PSE of the $\mathrm{S}_{1}$ order and $294 \mathrm{~g}$ for the value for the $S_{2}$ order. These estimates are too small by $11 \%$ and $12 \%$, respectively. In an experiment comparable to this one, but where the subjects used only one hand (Michels \& Helson, 1954), the predictions were in error only from $2 \%$ to $6 \%$ with $5 \%$ being a typical error. The larger error in prediction in the present study could be interpreted as meaning that the change from one to two hands contributed a space error corresponding to roughly $5 \%$, or about $15 \mathrm{~g}$. It's interesting to ask where this difference arises. Is it inherent in using both hands as opposed to one? Is it a function of the degree of spatial separation? Does it simply represent the absence of some appropriate control condition?

\section{Judgment Mode}

The mean PSE for S-mode judgments was $306 \mathrm{~g}$; the mean PSE for V-mode judgments was $313 \mathrm{~g}$. This difference is only marginally reliable $(\mathrm{p}<.100$, Table 2). Evidently, it doesn't really matter whether the subject is asked to judge the variable against the standard or vice versa. Given a lack of empirical support, it isn't useful to explain the differential effect of presentation order as really being the result of a change in judgment mode. It appears that the differential effect is caused solely by having the standard occur after the variable stimulus instead of before.

In this way, this negative finding is satisfying, for now a whole literature based upon an accidental confounding of variables doesn't have to be reinterpreted. Nor do we have to feel foolish for unthinkingly following three generations of authors who provided us with the confounded technique. In another vein, the negative result does provide some support for the common practice of transforming $\mathrm{V}$-mode judgments into $\mathrm{S}$-mode judgments when the results of the two presentation orders are combined. No additional effect or error beyond that attributable to the presentation order is being added to the data when these data are combined.

Still a nugget remains. The subjects must use the standard and variable stimuli differently in arriving at a judgment because presentation order has an effect. The results of this study simply and concisely indicate 
that there is no evidence that the cause is in terms of the different judgment modes which have typically been used.

\section{Miscellaneous Results}

Neither the hand the subject used to lift the standard (4 g difference in the mean PSE) nor the sex of the subject ( $2 \mathrm{~g}$ difference in the mean PSE) produced systematic effects on the PSE. The experimenter effect produced an $F$ ratio significantly less than one $(1 \mathrm{~g}$ difference in the mean PSE, Table 2). The usual interpretation of $F$ ratios smaller than one is that some nonrandom and unspecified variable was operating in addition to the many unspecitied random variables assumed in the analysis of variance model (Bennett \& Franklin, 1954). Perhaps this finding is simply showing that the two experimenters, by continually comparing their procedural techniques throughout the experiment. were able to minimize the differential effects they had upon the subjects.

The reliable interaction of presentation order with the left or right hand lifting the standard (A by $\mathrm{C}$ ) has been broken down into two orthogonal contrasts. The $A_{0}$ by $C$ component which is not reliable checks on the possibility of a differential effect on the PSEs of the two successively presented conditions. The $A_{1}$ by $C$ component of the total interaction contains almost all of the variance. This component is the comparison of the mean of the $S_{1}$ and $S_{2}$ presentation orders vs. the $S_{0}$ presentation order with the effect taken separately for the standard in the left hand and the standard in the right hand. For the $S_{1}$ and $S_{2}$ orders, the PSE for the standard lifted by the right hand is larger. For the $S_{o}$ presentations, the PSE for the standard in the left hand is larger. The cause of this difference can be traced by examining the marginally significant three-way interaction of presentation order, judgment mode, and hand lifting the standard (A by B by $\mathrm{C}$ ). Almost all of the variance attributed to this interaction is contained in the component labeled $A_{1}$ by $B$ by $C$. For the $S$ mode of judgment, the PSEs are about the same value for the standard on the left and for the standard on the right. This is true for all three presentation orders. The interaction occurs when the PSEs for the $V$ mode of judgment are considered. For the $S_{1}$ and $S_{2}$ orders, the values for the standard on the right are larger. But for the $S_{O}$ presentations, the PSE for the judgments when the standard is on the left is larger. The pattern for the $S_{1}$ and $S_{2}$ orders would be duplicated in the $S_{0}$ order if the group of subjects who were presented with the standard on the left judge left in terms of right had not been deviant. Perusal of the PSEs for the individual subjects within this group shows two of the eight with a PSE in excess of $370 \mathrm{~g}$. While these are not the largest PSEs obtained in the entire experiment, they are much larger than those of the remaining six subjects in that group. The individual PSEs for that group were 301 , $312,315,317,324,338,373$, and $373 \mathrm{~g}$.

The subjects asked to judge the simultaneously presented stimuli did not have the powerful cue of successive stimulus presentation to help them remember their task. They had to remember to judge the right stimulus in terms of the left or the left in terms of the right. They could not use the cue of judging first in terms of second, or second in terms of tirst. If the subjects became confused on some trials, and reversed the judgment order, then the judgment curve would have been more horizontal for these subjects and their PSE would be less reliable.

As a check of this possibility, the protocols of the two subjects with the exceptionally large PSEs were examined. At the time they were run, notations were made which suggest that the judgments may have been reversed on a couple of trials but that the tendency for reversal was corrected. If the mean of the remaining six subjects replaces the PSEs of these two subjects, the significant $A$ by $B$ by $C$ and $A$ by $C$ interactions disappear without, incidentally, causing the $B$ by $C$ interaction to become significant. Since the significant interactions do disappear when the data of the two deviant subjects-who may not have followed instructions-is removed, probably not too much should be made of them.

If, for the $S_{0}$ presentations, certain of the PSEs were spuriously too large or small because subjects did not judge the weights consistently in one mode. then perhaps the variability of these groups is larger than for the groups of subjects judging the stimuli for the $S_{1}$ and $S_{2}$ presentation orders. A Bartlett's test for homogeneity of variance (McNemar, 1969) was performed on the standard deviations of the 12 groups (see Table 1 for the values). The results did not indicate that the variability of the groups differed $\left[\chi^{2}(11)=15.933, p>.100\right]$.

The interaction of judgment mode with the hand lifting the standard ( $\mathrm{B}$ by $\mathrm{C}$ ) details the reliability of the effects for judging the right stimulus in terms of the left $(312 \mathrm{~g})$ vs. judging the left stimulus in terms of the right $(307 \mathrm{~g})$. This judgment variable was also nonsigniticant.

Because the main effect of experimenter and sex of the subject were not reliable, all interactions containing either of these effects were pooled. The pooled interaction term was not significant.

\section{Summary of Results and Conclusions}

In this study, the only design variable which systematically influenced the size of the PSE was whether the standard stimulus was presented before, with, or after the variable stimulus. Using the results of simultaneous presentations as a reference point. there is a different effect on PSE of presenting the 
standard before the variable than presenting it after the variable. This result implies that the subjects differentiate between the standard and variable stimuli as they make their judgments. Since, in this type of experiment, the subjects generally are not told how many stimuli will be used or that they'll be using one particular stimulus intensity as a reference or standard, the results are particularly intriguing. The effect cannot be explained in terms of the judgment task. It is of little importance whether the subjects are judging the variable against the standard or vice versa. What is important is whether the standard is the first or second weight of the pair to be lifted.

\section{REFERENCES}

Bennett, C. A., \& Franklin, N. L. Statistical analysis in chemistry and the chemical industry. New York: Wiley, 1954.

EDWARDs, A. L. Experimental design in psychological research (Rev. ed.). New York: Holt, Rinehart and Winston, 1964.

FECHNER, G. Elements of psychophysics (H. E. Adler, Trans.). New York: Holt, Rinehart and Winston, 1966. (Originally published, 1860.)

Guilford, J. P. Psychometric methods (2nd ed.). New York: McGraw-Hill, 1954.

Helson, H. Adaptation-level theory: An experimental and systematic approach to behavior. New York: Harper \& Row, 1964.
Helson, H., \& Himmelstein, P. A short method for calculating the adaptation-level for absolute and comparative rating judgments. American Journal of Psychology, 1955, 68, 631-637.

Helson, H., Michels, W. C., \& Sturgeon, A. The use of comparative rating scales for the evaluation of psychophysical data. American Journal of Psychology, 1954, 67, 321-326.

McNemar, Q. Psychological statistics (4th ed.). New York: Wiley, 1969.

Michels, W. C., \& Helson, H. A reformulation of the Fechner law in terms of adaptation-level applied to rating-scale data. American Journal of Psychology, 1949, 62, 355-368.

Michels, W. C., \& Helson, H. A quantitative theory of timeorder effects. American Journal of Psychology, 1954, 67, 327-334.

NeEdham, J. G. The time-error in comparison judgments. Psychological Bulletin, 1934, 31, 229-243.

\section{NOTE}

1. Unfortunately, the terminology at this point becomes irrational. When we change our procedures to eliminate space and time errors, a significant constant error usually remains. This constant error, which has come to be known as the time-order error or the time-order effect, is the phenomenon of decentered judgments which Helson's adaptation-level theory states is a basic quality of judgment.

(Received for publication February 12, 1975; revision received April 28, 1975.) 